\title{
External Approach for the Treatment of the Aging Nasal Tip
}

\author{
Melissa Hu
}

\section{ABSTRACT}

Changes seen in the nose from increased skin elasticity, weakened nasal cartilages, and detachment of ligaments can lead to cosmetic and functional impairment. Esthetically, the nasal tip droops, the nose lengthens, the columella shortens, and the ala collapse. Functionally, the internal and external nasal valves weaken, the anterior nares narrow, and airway turbulence increases, thus contributing to the effect of nasal obstruction. Skin redundancy of the elderly nose may compromise outcomes when typical rhinoplasty techniques are applied. Herein is described a technique pioneered by Fred Stucker, involving a direct external approach to address the aging nasal tip. The technique can be performed under local anesthesia and in combination with other procedures for the aging nose, such as rhinophyma excision or septoplasty for the aging nose.

Keywords: Aging nose, External approach, Nasal tip ptosis, Rhinolift.

How to cite this article: Hu M. External Approach for the Treatment of the Aging Nasal Tip. Int J Head Neck Surg 2016;7(3):165-167.

Source of support: Nil

Conflict of interest: None

\section{INTRODUCTION}

Characteristic changes of the aging nose include nasal tip ptosis, lengthening of the nose, pseudohump formation, and thick sebaceous skin. Much like skin in other areas of the face, the nasal skin loses elasticity over time. The skin may also thicken and becomes more sebaceous. The weight of this skin places further stress on the already weakening nasal tip support mechanisms. The most notable change with age is the weakening and separation of the fibrous attachments between the upper lateral cartilages (ULCs) and the lower lateral cartilages (LLCs). ${ }^{1,2}$ Cumulative with other changes including weakening of nasal cartilages, maxillary retrusion with atrophy of muscle, bone, and fat, the nasal tip begins to droop and the internal nasal valve weakens.

Cosmetically, the aging nose results in nasal lengthening, deprojection, and derotation of the nasal tip. ${ }^{3}$

Private Practitioner

Clear Lake ENT, Houston, Texas, USA

Corresponding Author: Melissa Hu, Private Practitioner, Clear Lake ENT, Houston, Texas, USA, Phone: +2813357755 e-mail: melissahu80@gmail.com
The ideal nasolabial angle is described to be $95^{\circ}$ to $100^{\circ}$ in men. Ptotic noses will have a more acute nasolabial angle and subsequently have the appearance of a long nose. With nasal tip ptosis, the columella shortens, the supratip crease becomes more prominent, and a dorsal pseudohump appears. 3,4

Functionally, the ptotic nose may lead to internal and external nasal valve compromise. The attachments between the ULCs and LLCs loosen, causing collapse of the internal nasal valve, particularly on inspiration. The gradual descent of the nasal tip, shortening of the columella, and weakening of the alar cartilages may also lead to narrowing of the external nasal valve. These changes increase nasal airway resistance, and lead to nasal obstruction with breathing.

The ptotic nose can be diagnosed by a simple maneuver. The patient's nasal tip is manually elevated and rotated superiorly. If the patient reports subjective improvement in nasal breathing, and the appearance of a dorsal hump is mitigated, the patient is diagnosed with nasal ptosis and would likely benefit from a rhinolift procedure. ${ }^{4}$

The procedure described in this article is pioneered by Fred Stucker. Experience with treating skin malignancies along the nasal dorsum led him to the incidental discovery that nasal breathing improves with the horizontal excision and primary closure of skin in the supratip region. Stucker gradually modified this technique to include suspension of the LLC to the ULC through this external approach. This, in effect, functionally reinforces the internal nasal valve and improves nasal breathing in the weakened, ptotic nasal tip.

\section{TECHNIQUE}

The technique described was introduced by Fred Stucker. The procedure may be performed under local anesthesia or under general anesthesia in combination with other procedures, i.e., septoplasty, rhinoplasty, and rhinophyma excision. A horizontal ellipse, on average $1 \times 3 \mathrm{~cm}$ in dimension, is drawn across the center of the supratip crease. The supratip crease is identified with elevation and superior rotation of the nasal tip. The shape of the skin excision may be modified to account for any nasal tip asymmetries. The skin and subcutaneous tissues are then infiltrated with lidocaine $1 \%$ with epinephrine 1:100,000. The patient is the prepped in the typical sterile fashion. The skin is then incised down to superficial muscular aponeurotic 
system fascia, beveling the incision inwards to assist with skin eversion at the time of closure. Full-thickness skin is removed. Sharp dissection is then performed to reveal the cephalic border of the LLCs and the caudal border of the ULCs. In the aging patient, there is typically disarticulation of the intercartilagenous junction with downward displacement of the LLC; 4-0 polydiaxanone mattress sutures are placed approximately $1 / 3$ to $1 / 2$ below the cephalic border of the LLC and suspended $1 / 4$ to $1 / 3$ above the caudal border of the ULC. One mattress suture is placed on each side before being tied down sequentially. Tension is applied to the suture until the optimal position for elevation of the nasal tip, and suspension of the internal nasal valve is achieved. The suture is then tightened to slightly overcorrect the nasal tip ptosis. A second set of mattress sutures may be applied in order to fine tune suspension of the internal and external valve regions or address any asymmetries of the nasal tip. The skin is then closed with 5-0 chromic in a running locking fashion.

\section{DISCUSSION}

Nasal tip ptosis is largely due to the increased laxity of the fibrous attachments between the ULCs and the LLCs. Other causes for nasal tip ptosis include a long septum, heavy nasal skin, an overactive depressor septi muscle, an elongated LLC that rotates the nasal tip caudally, vertically oriented lateral crura, or short and weak medial crura. ${ }^{2}$ Several techniques and algorithms have been described to treat the aging nasal tip which include columellar and lateral crural strut grafts, spreader grafts, nasal tip grafts, cartilage excisions of the caudal septal and lateral crura, nasal tip suture techniques, and skin excisions at the rhinion. ${ }^{4-6}$ Studies have also demonstrated the effectiveness of suspension sutures of the
LLC to a more superior point on the ULC. This restores the normal anatomic relationship between the structures, and the ULC may further assist in stenting the vestibule open to improve nasal breathing. ${ }^{7,8}$

However, often the most difficult aspect of rhinoplasty in the aging patient is management of the excess nasal skin. The skin loses intrinsic elasticity, and with heavy thick nasal skin, it may not adapt to underlying cartilaginous and bony modifications in rhinoplasty procedures of aging patients. ${ }^{7,9}$ Several methods have been adopted to address this problem, from steroid injections to the supratip region, defatting of the subcutaneous tissues, and excisions from the dorsal skin. ${ }^{3,10}$

The external approach to nasal tip ptosis allows direct access to the junction of ULC and LLC and modification to reinforce or reconstruct the attachments. Previous descriptions of rhinolift procedures place the incision at the nasal root or glabellar region. ${ }^{7,911}$ This addresses skin elasticity with direct excision and allows the skin envelope to be pulled up in a pants and suspenders effect. However, this does not address the issues of weakened cartilaginous attachments or malrotated LLCs contributing to nasal ptosis. The direct excision of skin in the supratip region directly treats the inelastic, thick, and redundant skin of aging male noses and accesses the intercartilagenous junction for reinforcement. This approach may also be used for more extensive procedures. Ozturan et $\mathrm{al}^{12}$ applied a wide-open dorsal approach for ptotic noses to complete bone reduction, septoplasty, reconstruction of the nasal framework, and nasal tip work.

The technique described by Stucker is simple, quick, and effective. This procedure can easily be performed under local anesthesia in the clinic. The external scar is minimal when performed correctly (Figs 1A and B).

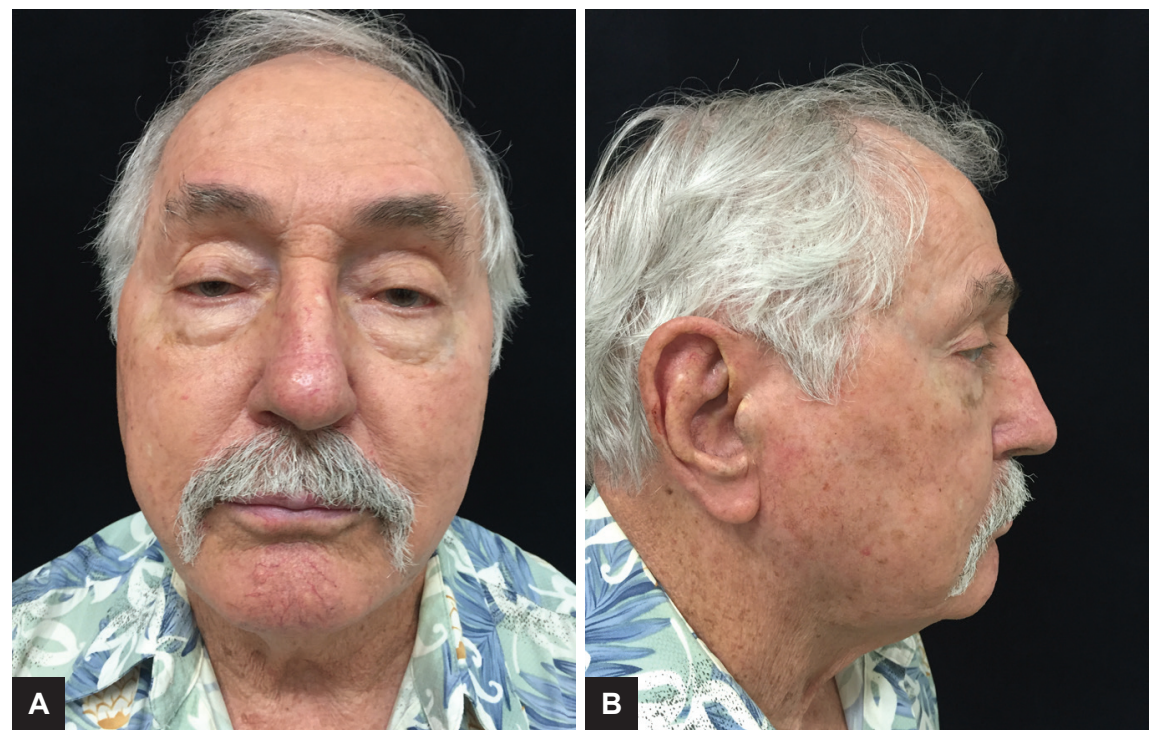

Figs 1A and B: (A) Frontal view of patient 7 months postoperative from external rhinolift; and (B) profile view of patient 7 months postoperative from external rhinolift 
With the mature patient, the surgeon must consider the medical comorbidities, health, and safety of performing rhinoplasty even for functional purposes. Over the 30 years that this procedure has been performed, we have found the functional and cosmetic changes to be long lasting in patients.

\section{CONCLUSION}

The aging nose is characterized by nasal tip ptosis. The technique described by Stucker addresses the specific anatomic changes with the aging nose, and corrects not only the cosmetic deformity of the nasal tip but also the internal and external nasal valve dysfunction. We found that most elderly patients are willing to trade a minimally perceptible external scar for significant cosmetic and functional improvement of the nasal tip and breathing respectively.

\section{REFERENCES}

1. Cochran CS, Ducic Y, DeFatta RJ. Restorative rhinoplasty in the aging patient. Laryngoscope 2007 May;117(5):803-807.

2. Sajjadian A, Guyuron B. An algorithm for treatment of the drooping nose. Aesthet Surg J 2009 May-Jun;29(3):199-206.
3. Quatela VC, Pearson JM. Management of the aging nose. Facial Plast Surg 2009 Nov;25(4):215-221.

4. Stupak HD, Johnson CM Jr. Rhinoplasty for the aging nose. Ear Nose Throat J 2006 Mar;85(3):154-155.

5. Rohrich RJ, Hollier LH Jr, Janis JE, Kim J. Rhinoplasty with advancing age. Plast Reconstr Surg 2004 Dec;114(7): 1936-1944.

6. Toriumi DM, Rosenberger E. Rhinoplasty of the aging nose. Facial Plast Surg 2016 Feb;32(1):59-69.

7. Slavit DH, Lipton RJ, Kern EB, McCaffrey TV. Rhinolift operation in the treatment of the aging nose. Otolaryngol Head Neck Surg 1990 Sep;103(3):462-467.

8. Zuliani GF, Silver WE. Analysis of nasal ptosis correction using lower lateral to upper lateral cartilage suspension. Arch Facial Plast Surg 2011 Jan-Feb;13(1):26-30.

9. Kabaker SS. An adjunctive technique to rhinoplasty of the aging nose. Head Neck Surg 1980 Mar-Apr;2(4):276-281.

10. Lemperle G, Biewener A. External skin excision in the sebaceous nose and supratip deformity. Aesthetic Plast Surg 1992 Fall;16(4):303-307.

11. Caterson SA, Singh M, Kueckelhaus M, Caterson EJ, Eriksson E. Skin excision as an adjunctive technique to rhinoplasty in middle-aged and elderly patients. Plast Reconstr Surg Glob Open 2015 Oct 9;3(10):e532.

12. Ozturan O, Ozucer B, Aksoy F, Tugrul S. Wide-open dorsal approach rhinoplasty for droopy noses. Aesthetic Plast Surg 2015 Feb;39(1):25-35. 